\title{
ENSAYO
}

\section{Medicina basada en la evidencia: una aproximación epistemológica}

\author{
Daniel Eduardo Henao', Fabián Alberto Jaimes² \\ 1 Grupo Reproducción, Universidad de Antioquia, Medellín, Colombia \\ 2 Grupo Académico de Epidemiología Clínica (GRAEPIC), Universidad de Antioquia, Medellín, Colombia
}

La propuesta de la medicina basada en la evidencia conjuga la experiencia del clínico con el análisis juicioso de los resultados de investigaciones clínicas de excelente calidad metodológica para la toma de decisiones médicas. Esta propuesta ha sido ampliamente divulgada por los líderes de opinión médica. Sin embargo, contrario al gran número de publicaciones que promueven esta práctica, no se evidencia en la literatura médica un espacio de discusión acerca de las implicaciones epistemológicas que ha tenido la implementación de esta práctica en el acontecer cotidiano del acto médico. La propuesta novedosa de la medicina basada en la evidencia consiste en priorizar las decisiones médicas de acuerdo con la disponibilidad del conocimiento como resultado de los estudios científicos.

Presentamos en este ensayo algunas reflexiones sobre la inconveniencia de la importación "ciega" del método científico de las ciencias exactas a la ciencia clínica, que es la principal fuente de evidencia para el médico, además de lo inadecuado de definir los estados de salud y enfermedad como continuos numéricos homogenizados por una escala de medición. Finalmente, proponemos que el reconocimiento del ser humano enfermo como sistema complejo, único e irrepetible, definido a su vez por su devenir y su contexto, nos obliga a reconocer que la propuesta de la medicina basada en la evidencia no es universal ni absoluta. Sólo en la medida en que dichas particularidades se tengan en cuenta, estaremos en capacidad de brindar una atención más idónea a nuestros pacientes.

Palabras clave: medicina basada en evidencia, epidemiología, conocimiento, ciencia.

\section{Evidence-based medicine: an epistemological approach}

Evidence-based medicine gathers physician's experience and the best scientific evidence to make medical decisions. This proposal has been widely promulgated by medical opinion leaders. Despite a large literature supporting this practice, a formal discussion has not been established regarding its epistemological consequences in daily medical work. The main proposal of evidence-based medicine consists of choosing the best medical decision according to the best available results from scientific studies. Herein, the goal was to highlight inappropriate application of the scientific method used by physics to clinical science. The inaccuracy resides in describing health and disease in strictly numeric equivalents that can be homogenized on a continuous scale. Finally, the authors consider each diseased human being as a complex system, unique and particular, and that this being is defined by an historical background as well as current actual context. Therefore, evidence-based medicine possesses certain limitations that must be recognized in order to to provide better health care to patients.

Key words: evidence-based medicine, epidemiology, knowledge, science.

\author{
Correspondencia: \\ Daniel Eduardo Henao, Carrera 53 № 61-30, Sede de \\ Investigación Universitaria, Universidad de Antioquia, \\ Medellín, Colombia. \\ Teléfono: (574) 219 6685; fax: (574) 2196470 \\ dhenao@medicina.udea.edu.co
}

Recibido:16/06/08; aceptado:13/11/08 
El acto médico se encuentra en la encrucijada de varias disciplinas científicas. La medicina se ha nutrido tradicionalmente del conocimiento producto de ciencias como la biología, la química y la física, para apoyar las decisiones clínicas respecto al cuidado de los enfermos. Aunque es clara la relación que existe entre la medicina y las ciencias denominadas "exactas" por su carácter cuantitativo, es también innegable cada acto médico se desarrolla en un contexto sociocultural. Lo anterior dota a esta actividad humana de un profundo contenido sociológico y le da validez a aquella expresión que afirma que la medicina es arte y ciencia.

El modelo médico tradicional resulta del análisis juicioso de la enfermedad de cada paciente que, en el primer momento, utiliza el conocimiento de la ciencia biomédica para determinar el diagnóstico del enfermo y las causas fisiopatológicas que subyacen al mismo, y posteriormente, procede a tomar una decisión terapéutica que sea consecuente con el diagnóstico realizado. Si bien la medicina y el quehacer de un médico están soportados en un cuerpo de conocimientos provenientes de las ciencias básicas biomédicas, desde hace algún tiempo se ha introducido un debate sobre la necesidad de una ciencia médica "propia" (1); esto es, de una disciplina científica que tenga que ver necesariamente con los problemas a los que un médico se ve enfrentado en su práctica cotidiana, ya que frecuentemente dichos problemas escapan al alcance del conocimiento básico producto de esas otras ciencias. Respecto a este debate, ha surgido la medicina basada en la evidencia como una respuesta a las necesidades de los médicos de contar con conocimientos derivados de una disciplina científica directamente relacionada con su quehacer diario (1).

En un apasionado artículo publicado en 1992 en el Journal of the American Medical Association (1) por el denominado Evidence-Based Medicine Working Goroup de McMaster University en Canadá, se esbozaron los planteamientos de lo que los autores pronosticaron sería un nuevo paradigma en la práctica clínica.

En palabras de uno de sus impulsores más importantes "la medicina basada en la evidencia es el uso juicioso, explícito y consciente de la mejor evidencia disponible para el cuidado del paciente individual. Su práctica integra la experiencia clínica personal con la mejor evidencia clínica proveniente de estudios clínicos de la mejor calidad metodológica" (2).

Estas nociones resultan en una interesante propuesta para los clínicos en su ejercicio diario. Sin embargo, la introducción de una nueva forma de llevar a cabo el ejercicio médico resulta en un punto de inflexión respecto a lo tradicionalmente planteado (3), lo cual genera serias resistencias por parte de aquéllos que encuentran en la manera tradicional la forma idónea de cuidar a los enfermos.

Debe anotarse que el surgimiento de la medicina basada en la evidencia no es fortuito ni responde a la inspiración genial de un grupo de sujetos. Su aparición está soportada por un contexto social que permitió e influenció sus planteamientos principales. La última década del siglo pasado fue testigo de numerosos avances científicos, entre los que se cuentan los de las denominadas ciencias duras. Así, no es de extrañar que la práctica médica se dejara contagiar por el agitado impulso de la formulación de leyes matemáticas para explicar los fenómenos naturales. Claramente, este "contagio" se observa cuando se propone que un componente principal y una diferencia fundamental entre el modelo médico tradicional y la medicina basada en la evidencia es que esta última provee al médico herramientas matemáticas que le permiten explicar situaciones clínicas cotidianas de manera más precisa (4). Por ejemplo, hoy se puede afirmar con cifras la probabilidad de eventos convulsivos futuros en un paciente de 40 años previamente sano quien presenta un episodio de convulsiones tónico-clónicas generalizadas por primera vez. Esta capacidad, que ofrece la medicina basada en la evidencia, proviene del análisis juicioso de varios estudios clínicos. Por el contrario, con el modelo médico clásico, no hubiera sido posible afirmar esto numéricamente ya que la decisión acerca del pronóstico del paciente hubiera estado sustentada en la experiencia del 
médico tratante y en la eventual opinión de un reconocido experto en el tema.

Uno de los pilares fundamentales en los que está cimentada la medicina basada en la evidencia es la evidencia clínica externa. Es decir, los estudios clínicos experimentales se convierten en una invaluable fuente de información para la práctica clínica. Sin embargo, surge la inquietud acerca de qué hacer cuando no se pueden llevar a cabo experimentos clínicos, sea por cuestiones éticas o limitaciones puramente técnicas, para solucionar preguntas clínicas relevantes. El uso de la "mejor evidencia disponible" referida única y exclusivamente a los ensayos clínicos con asignación aleatoria, es una de las principales limitaciones planteadas a la medicina basada en la evidencia; se argumenta, entre otras razones, que la evidencia revelada en estos estudios se hace con base en grupos de pacientes y no especifica o no permite precisar con exactitud la aplicación de los resultados a los pacientes individuales (5).

Porotraparte, los ensayos clínicos con asignación aleatoria generalmente responden a preguntas de tratamiento y, por lo tanto, se ofrece evidencia mucho más limitada para otras preguntas clínicas relevantes como las de diagnóstico, pronóstico y causalidad (6). La propuesta de usar el método científico para sustentar las decisiones terapéuticas de un paciente ha sido una premisa irrefutable para la medicina occidental, por lo que los planteamientos de la medicina basada en la evidencia han sido bien recibidos por la comunidad médica en general. No obstante, las principales críticas de este modelo se han enfocado en la descalificación por parte de sus seguidores del modelo médico tradicional (7) o en la dudosa intención científica de algunos investigadores que llevan a cabo ensayos clínicos con asignación aleatoria con fines puramente lucrativos (8). Ocasionalmente, las críticas se refieren a las limitaciones metodológicas inherentes a la propuesta (9) pero, a pesar de la extensa correspondencia (10-18), no se ha planteado formalmente una discusión acerca de los alcances epistemológicos de la medicina basada en la evidencia. Lejos de radicalizar el discurso acerca de si es buena o mala, ni mucho menos tratar de sentar posiciones irreconciliables acerca de la misma, debe hacerse un debate juicioso sobre las bondades y limitaciones que ofrece esta nueva propuesta $y$, por supuesto, debe reconocerse su papel en el contexto de los avances hechos por el modelo médico tradicional. Probablemente, de la armoniosa convivencia de ambos modelos emergerán herramientas valiosas para el cuidado de los enfermos.

Una pregunta fundamental después del advenimiento de la medicina basada en la evidencia es acerca de la implementación y aceptación de este modelo en la práctica clínica cotidiana. A este respecto, se han llevado a cabo estudios descriptivos $(19,20)$ en los cuales se ha demostrado que más del $80 \%$ de los tratamientos suministrados a los pacientes admitidos a unidades médicas universitarias y suburbanas están basados en evidencias provenientes de ensayos clínicos con asignación aleatoria o, en su defecto, de otro tipo de estudios clínicos de naturaleza no experimental.

Aunque los resultados de estos estudios suscitaron controversias respecto a su diseño metodológico $(21,22)$, nunca se planteó por parte de los autores ni de los lectores el verdadero impacto clínico que tuvieron los tratamientos basados en la evidencia; es decir, nunca se cuestionó si en la vida real de la práctica cotidiana el desenlace clínico de los pacientes que recibieron tratamiento basado en evidencia científica, al igual que en los pacientes de los ensayos clínicos con asignación aleatoria, era mejor que el de aquéllos cuyo tratamiento no estaba sustentado en resultados derivados de la evidencia experimental.

La medicina basada en la evidencia nos pone de manifiesto una oportunidad valiosa para dilucidar problemas médicos cotidianos mediante el método científico; sin embargo, cuando no se reconocen las limitaciones inherentes a la complejidad biológica-social de los pacientes y las limitaciones de la supuesta validez universal del método científico, se puede caer en el desafortunado evento de considerar y ejercer esta práctica como un absurdo dogma. 
En este ensayo se hace una reflexión crítica sobre el quehacer del investigador y sus métodos, y de este modo se pretende reconocer las limitaciones propias del método de la medicina basada en la evidencia. Se discuten las siguientes consideraciones: la influencia de los métodos utilizados en la física y la química sobre la investigación biomédica básica y aplicada, y las profundas implicaciones que esto ha tenido en el desarrollo y la producción de conocimiento; las implicaciones de los conceptos de lo normal y lo patológico o de la salud y la enfermedad utilizados frecuentemente en el quehacer del investigador clínico, y las consecuencias que éstos tienen sobre la terapéutica practicada por el médico cotidianamente. Y, finalmente, algunas conclusiones de las posibles repercusiones que puedan tener los dos puntos anteriormente planteados sobre la medicina basada en la evidencia propiamente dicha y sobre el acontecer terapéutico ordinario de un médico tratante.

\section{Importación del modelo empírico-analítico} tradicional a la práctica médica

¿Pueden los fenómenos biológicos tratarse matemáticamente? ¿Existen leyes que expresen matemáticamente los fenómenos biológicos y clínicos relacionados con los pacientes?

Según Bichat (23), la inestabilidad e irregularidad son características de los fenómenos vitales; por lo tanto, hacerlos entrar por la fuerza dentro del marco rígido de las relaciones métricas significa desnaturalizarlos. Reconocer la complejidad inherente al objeto de estudio de la biología y de la medicina basada en la evidencia en particular, es un punto de partida para un uso más idóneo de la misma en la práctica clínica.

Sin duda alguna, el cuerpo de conocimientos derivado del método empírico-analítico ha sido excepcional en la última mitad del siglo XX, y podríamos afirmar que la física y la química lograron dar un paso adelante respecto al conocimiento del comportamiento de la materia inerte. Del mismo modo, la biología molecular ha tenido avances considerables en relación con el entendimiento de los seres vivos. Así, la formulación matemática de las leyes que dieron cuenta de los fenómenos observados experimentalmente se constituyó como el gran logro del método científico durante el siglo XX.

Sin embargo, otro logro significativo del método científico durante el pasado siglo fue el reconocimiento de la incertidumbre y la no linealidad del comportamiento de la materia viva respecto al contexto donde se desenvuelve. La introducción y el reconocimiento del contexto es fundamental, ya que supedita las leyes que determinan el comportamiento de la vida al medio donde ella se organiza, lo que obliga a reconocer que las múltiples formas de relación de la materia viva con el contexto donde se desenvuelve le permiten desplegar variados patrones de comportamiento durante su devenir $y$, por lo tanto, la reproducibilidad planteada por la ciencia clásica no es posible en todos los casos. De lo anterior se desprende que la incertidumbre es inherente al objeto de estudio y no es un problema que deba ser resuelto por el método mismo.

La optimización de las formas de medición no garantiza el comportamiento lineal y predecible del fenómeno estudiado. Los planteamientos de la mecánica cuántica son el reflejo de la preocupacióndelosfísicossobreestasconsideraciones, que tiene implicaciones importantes para la biología y la ciencia clínica. La multiplicidad de las relaciones, de sus componentes, los múltiples patrones de evolución y la sensibilidad a las condiciones iniciales, son características comunes a los sistemas complejos (24), entre los cuales se inscribe la materia viva. Por lo tanto, el estudio sistemático de lo humano, aun del ser humano enfermo, debe tener en cuenta esto. El humano, como sistema complejo, opera como una totalidad incluyente que responde congruentemente a los cambios del contexto que éste le propone y está lejos de enmarcarse dentro de un escrutable cuerpo de leyes universales, rígidas e inmodificables.

Por el contrario, la armoniosa relación del ser vivo con su entorno depende de las variadas y novedosas formas de respuesta que éste posee. Así, pues, la no universalidad de las leyes físicas y la complejidad inherente a la materia viva y, en consecuencia, al ser humano, están lejos de ser 
meras críticas eruditas y deben ser elementos indispensables que se deben tener en cuenta cuando se realiza investigación biomédica $y$, más aún, investigación clínica. Por lo tanto, la formulación matemática de fenómenos biológicos y clínicos debe estar supeditada al contexto donde se originaron y así deben ser interpretados.

El conocimiento derivado de las investigaciones es muy útil para la práctica; sin embargo, los términos cuantitativos y estadísticos producto de los estudios clínicos no determinan completamente el contexto clínico del paciente; y la condición de enfermedad y la necesidad terapéutica no dependen exclusivamente de las mediciones métricas, aunque valiosas y necesarias, sino del análisis juicioso que no excluya los factores biológicos, psicológicos y sociales del paciente que no siempre pueden cuantificarse. Resulta tan equivocado afirmar que la experimentación clínica es innecesaria como que el cuidado de los pacientes depende únicamente de un conjunto de leyes invariables y fuera de contexto. En este caso, es necesario reconocer que el alcance de la medicina basada en la evidencia posee ciertas limitaciones.

Para Comte (25), una experimentación cualquiera está destinada siempre a descubrir de acuerdo con qué leyes cada una de las influencias determinantes o modificadoras de un fenómeno participan en su realización; y en general, un experimento consiste en introducir en cada condición identificada un cambio bien definido, con el objeto de apreciar directamente la respectiva variación del fenómeno mismo.

Imaginemos, de esta forma, un experimento clínico en el cual se trata de comparar un grupo de pacientes en tratamiento con un medicamento antihipertensivo contra un grupo control tratado con placebo, respecto al desarrollo de complicaciones de la hipertensión arterial cuantificadas por el número de ingresos hospitalarios con este diagnóstico en cada grupo. Así tenemos, según Comte, un experimento que tratará de descubrir las leyes que determinan cómo la introducción de un medicamento puede modificar la historia natural o el curso clínico de una enfermedad. Un paso inicial para llevar a cabo dicha investigación es la elección de los pacientes, es decir, a quién considerar hipertenso y a quién no; y en este punto se determinan los criterios de inclusión que definirán la muestra para el experimento. Aquí está el primer escollo que debe resolver el investigador, ya que debe conocer o tener a la mano una herramienta que le permita discernir sobre la naturaleza de cada uno de los pacientes, que le permita conocer quién está enfermo y quién no. Por lo tanto, la pregunta por resolver es ¿qué es la hipertensión arterial?, ¿quiénes padecen de esta enfermedad? Para resolverla, actualmente se cuenta con la definición del Joint National Committe 7 (26) que dice que hipertenso es aquél que tenga una presión arterial mayor o igual a 140/90 mm Hg. Sin embargo, ¿todo aquel que tenga cifras de presión arterial por encima de este límite está necesariamente enfermo?, ¿la mera desviación de la norma expresada en términos numéricos explica la hipertensión arterial?

El aumento de la resistencia vascular periférica que se refleja clínicamente en cifras tensionales aumentadas, es necesario para que exista hipertensión arterial; sin embargo, un aumento de esta resistencia no es suficiente para explicar la hipertensión arterial como enfermedad. Este aumento en la resistencia vascular, y las consecuentes cifras de presión arterial elevadas, operan como factores desencadenantes de un proceso de organización sistémico que eventualmente resulta en lo que denominamos hipertensión arterial esencial. Por lo tanto, el aumento en las cifras de presión arterial es una causa necesaria pero no suficiente para el desarrollo de la enfermedad. Es fundamental tener en cuenta otros factores que confluyen para el desarrollo de la enfermedad y el cómo ésta se manifiesta clínicamente, así como conocer y reconocer los nuevos eventos biológicos que subyacen en el enfermo. La afirmación de que nuevos eventos ocurren en el enfermo nos centra en uno de los pilares fundamentales de esta discusión: el reconocimiento de la enfermedad como un comportamiento novedoso durante el devenir de un ser humano admite una diferencia cualitativa respecto al comportamiento normal 
$y$, por lo tanto, niega que estos eventos sean hechos continuos que se pueden representar simplemente como una homogeneidad numérica. Para ampliar esta discusión es necesario reflexionar sobre las nociones de salud y enfermedad o, de otra forma, acerca de lo normal y lo patológico.

Acerca de lo normal y lo patológico: implicaciones en la medicina basada en la evidencia

¿Es la enfermedad una alteración cuantitativa de la salud?

Esta preocupación no es reciente y es el argumento principal de la tesis doctoral del filósofo francés Georges Canguilhem titulada "Lo normal y lo patológico" (27). Las reflexiones generadas sobre este tema nos remontan a los principios planteados por Comte, quien tomando como referencia el modelo de irritación propuesto por Broussais, afirma:

"De acuerdo con el principio eminentemente filosófico que de ahora en adelante constituye la base general y directa de la patología positiva -y cuyo establecimiento definitivo debemos al genio audaz y perseverante de nuestro ilustre conciudadano, el señor Broussais-, el estado patológico no difiere en absoluto radicalmente del estado fisiológico, con respecto al cual sólo podría constituir, en cualquier aspecto, una mera prolongación más o menos extensa de los límites de variación ya sea inferiores ya superiores, propios de cada fenómeno del organismo normal sin producir nunca fenómenos verdaderamente nuevos que, en determinado grado, ya no tuvieran en absoluto análogos meramente fisiológicos" (23).

La homogeneidad que expresa en términos numéricos el continuo entre la salud y la enfermedad es el corolario del argumento planteado por Comte. No obstante, resulta llamativo cómo este filósofo nunca ilustró sus planteamientos con un caso médico real que permitiera materializar dicha proposición.

Dos objeciones surgen a partir del argumento anteriormente planteado. La primera es cómo se determina el comportamiento normal 0 , en nuestro caso particular, cómo se define el estado de salud. Supongamos que se quisiera determinar el comportamiento normal de la presión arterial en un grupo de hombres en edades entre 18 y 40 años, residentes en un país industrializado, previamente sanos y que asisten a un chequeo médico laboral de rutina. Estos pacientes se ingresan en un programa de seguimiento para determinar cuál es el comportamiento normal de sus cifras de presión arterial. Después de dos años de seguimiento periódico se logra establecer el valor promedio de presión arterial para estos individuos. La interpretación inicial de este hallazgo podría ser que el resultado del comportamiento promedio de la presión arterial resulta en la norma para los hombres entre 18 y 40 años edad. Sin embargo, si reconocemos que el contexto es un factor determinante para el devenir de cualquier sistema, solamente podríamos decir que este valor sería válido para los sujetos que participan en dicho estudio.

En este sentido, es muy oportuna la reflexión que Claude Bernard hace respecto a la aversión que tiene a la expresión de todo resultado biológico analítico o experimental como promedio. Él afirma que la utilización de los promedios hace que desaparezca el carácter esencialmente oscilatorio y rítmico del fenómeno biológico funcional (28). Aunque la expresión del promedio de una variable fisiológica puede resultar útil si se utilizan las herramientas matemáticas adecuadas que tengan en cuenta las oscilaciones de éste, el problema de cuándo considerar un comportamiento anormal no queda resuelto.

Imaginemos que el resultado de dicho valor promedio de la presión arterial media es $90 \pm 10$ $\mathrm{mm} \mathrm{Hg}$, y que éste denota el comportamiento normal de esta población. Si en este grupo de población se encontrara una persona con cifras de presión arterial media mayor de $110 \mathrm{~mm} \mathrm{Hg}$ sin ninguna queja y aun si después de varios años de seguimiento registra cifras elevadas respecto a esta norma, ¿podríamos considerarlo enfermo o se necesita un contexto que soporte esta categoría?

El siguiente ejemplo, aunque coloquial, resulta ilustrador. Durante una cena dos amigos se 
comen en promedio 5 panes cada uno; por lo tanto, se supondría que ninguno de los dos tiene hambre. Pero esto puede resultar engañoso ya que uno de ellos se comió diez panes y el otro ninguno. Ambos están experimentando situaciones cualitativamente diferentes que los ponen en dos categorías que no pueden homogenizarse numéricamente y que, además, son novedosas. La saciedad no encuentra su definición en la ausencia de hambre, como la salud no lo hace con la ausencia de la enfermedad. Por lo tanto, la interpretación fuera de contexto de un valor numérico no indica una alteración de lo normal, ni mucho menos la presencia o ausencia de una enfermedad; es necesario interpretar otros factores influyentes que no son necesariamente de naturaleza cuantitativa. Luego, la definición de normal que no resuelve Comte en su premisa sobre la patología positiva no responde solamente a un valor numérico, ya que la desviación del promedio no necesariamente indica un comportamiento anormal o patológico.

Por otra parte, surge una segunda inquietud respecto al planteamiento mencionado por Comte. Si todos los fenómenos patológicos tienen su equivalente fisiológico, uno se atreve a preguntar qué tienen de similar un paciente con síndrome de inmunodeficiencia adquirida respecto a un paciente sano, ¿acaso la diferencia cuantitativa en el recuento de linfocitos CD4+ explica la impresionante e impredecible adaptación micro-biológica que sucede en los pacientes con esta enfermedad?

El desarrollo de otras enfermedades podría ilustrar esto. En el caso de la tuberculosis es bien conocido cómo la infección con Mycobaterium tuberculosis no garantiza la aparición de la enfermedad; es necesario que ocurra una nueva forma de organización sistémica que aparece a favor de la aparición de ésta. Cabe anotar que las nuevas formas de organización dependen del devenir propio del sistema y de innumerable cantidad de variables conocidas y desconocidas; por lo tanto, el efecto (aparición de la tuberculosis) desencadenado por el estímulo (presencia de $M$. tuberculosis) es impredecible. Es necesario que existan dos estados de organización diferentes que soporten la aparición o ausencia de la enfermedad.

Así, con las evidencias con las que se cuenta actualmente, se reconoce claramente cómo la enfermedad, lo patológico o lo anormal son hechos que se definen por sí mismos y no en términos de la salud, lo fisiológico y lo normal, respectivamente. Como hemos reconocido anteriormente, en su surgimiento los discursos científicos no están exentos de las influencias sociales. Hay que tener en cuenta que en el siglo XIX la fisiología ya era considerada como una ciencia; así, la prolongación de la patología como una extensión de ésta le brindaba herramientas científicas que les permitían a los médicos sustentar el equipamiento terapéutico.

Por el momento, se han dimensionado los aspectos que tienen que ver con el comportamiento complejo inherente a lo biológico y a la organización compleja que genera una enfermedad; sin embargo, para la referencia a la enfermedad se hace prioritario tener en cuenta lo humano propiamente. La salud, dice Leriche, es la vida en el silencio de los órganos y, por el contrario, la enfermedad es aquéllo que molesta a los hombres en el normal ejercicio de su vida y en sus ocupaciones y, sobre todo, aquéllo que los hace sufrir (29). Si miramos con detalle esta proposición, podríamos aun afirmar que la propuesta de Comte es insuficiente en cuanto que el dolor y el sufrimiento experimentados por una persona enferma no tienen equivalentes en la salud ni en lo normal. El reconocimiento de la enfermedad como una novedosa forma de organización biológica y el reconocimiento de la experiencia deletérea que ésta representa para aquél que esté enfermo, permitirán dimensionar al humano enfermo como tal y no como el resultado promedio de mediciones métricas.

\section{Consideraciones finales}

La medicina es una práctica humana que trata varios de los temas más trascendentales para el ser humano: la enfermedad y la muerte. En éstas se identifica el sufrimiento como común denominador y, por lo tanto, es comprensible que de esta práctica se ocupen discursos de diversa naturaleza. Al poner en contexto los 
planteamientos explicados en este escrito, es necesario reconocer que lo discutido está en relación exclusivamente con el desarrollo de la sociedad occidental, y consecuentemente, lo aquí planteado se circunscribe a su dinámica propia.

El conocimiento de la naturaleza, producto de la actividad de la ciencia y como discurso insigne de esta sociedad, ha resultado en progresos tecnológicos inimaginables. Los avances en telecomunicaciones, informática y medios de transporte son evidentes; sin embargo, los avances producidos respecto al entendimiento de otra clase de fenómenos, como la enfermedad, el dolor y las dinámicas sociales, no es tan evidente: ¿en qué radica tan abismal diferencia? El método científico utilizado para producir conocimiento es invariable independientemente del objeto estudiado; por esto, las limitaciones del conocimiento en los fenómenos mencionados parecieran estar en relación con las características de los mismos y no en el método, ya que éste es el mismo en todos los casos.

Hay que puntualizar que algunos fenómenos propios de la materia inerte son relativamente sencillos en términos de su dinámica lineal y reduccionista, pero lo contrario ocurre con los fenómenos propios de otras formas de organización de la materia. En la medida en que la materia va cambiando sus niveles de organización, las características de las interacciones que rigen su comportamiento se van haciendo más complejas. De esta forma, el comportamiento de un cuerpo inerte pero en movimiento puede predecirse con un margen de incertidumbre muy estrecho, mas no ocurre así con la dinámica celular o social.

Esporestoquedebellamarselaatención respecto a la pretensión de predicción y manipulación lograda con fenómenos naturales con niveles de organización simples, con respecto a fenómenos más complejos. No es suficiente ni apropiado utilizar el mismo método usado por la física y la química en el estudio de problemas clínicos. La formulación de leyes en física y química ha ayudado a dilucidar grandes problemas a la humanidad, pero hay que reconocer que el nivel de organización de la materia viva demanda una nueva formulación de "leyes" que tengan en cuenta la complejidad inherente a la vida misma y a lo humano. Por otra parte, el intentar permear los conceptos emanados de una disciplina exacta a otra cuyo objeto de conocimiento es más complejo debe mirarse con cautela; por lo que el lenguaje matemático utilizado por la física para la explicación precisa de sus fenómenos no debería convertirse en el objetivo más importante de las ciencias de la vida, ni de la medicina basada en la evidencia.

El reconocimiento de esta limitación de los métodos utilizados por la física y la química, para efectos de la comprensión de los fenómenos biológicos, debe alentarnos a indagar sobre las causas reales que generan el comportamiento caótico, impredecible y algunas veces desconcertante de los seres vivos, y muy especialmente del ser humano en condición de enfermedad. Si aceptamos que el enfermo es más que una alteración cuantitativa de la norma y reconocemos en éste un sistema biológico complejo que manifiesta su comportamiento sistémico cualitativamente diferente a través de lo que denominamos enfermedad, entenderemos las limitaciones reales de algunos experimentos clínicos.

El planteamiento inicial de la medicina basada en la evidencia como una novedosa herramienta para sustentar las decisiones clínicas en cada paciente no desconoce la habilidad ni la experiencia previa del clínico; por lo tanto, el análisis juicioso de los resultados de los estudios conjugado con la experiencia del médico sería lo ideal para una práctica médica idónea. Sin embargo, es necesario también reconocer y actuar como médicos sobre el caso bastante frecuente de las preguntas clínicas sin respuesta, o con respuestas insuficientes o inadecuadas, en la evidencia experimental de la investigación clínica.

Igualmente, se presume que las decisiones clínicas sustentadas en argumentos científicos deben resultar en mayores y mejores beneficios para los pacientes; sin embargo, y siguiendo la 
misma lógica planteada por la medicina basada en la evidencia, esto no se ha demostrado por medio de la evidencia empírica que brinda una investigación clínica. Por lo tanto, la justificación de invertir esfuerzo y recurso humano en estudios que pretenden resolver preguntas clínicas, está basada en un supuesto especulativo y no en un argumento científico.

Con los planteamientos en este escrito queremos suscitar este interrogante y advertir acerca de la necesidad de responderlo. El acto médico requiere un ejercicio intelectual demandante, en el cual el profesional debe tener en cuenta tres principios rectores: que su experiencia le brinda herramientas muy valiosas en el abordaje de los problemas clínicos, que existe un cuerpo conceptual proveniente del discurso de la medicina basada en la evidencia acerca de las pruebas disponibles para resolver esos problemas, y que las falencias inherentes a los dos anteriores pasan por el hecho irrefutable de que interactuamos con seres humanos únicos, particulares e irrepetibles. Finalmente, todo esfuerzo merece la pena cuando se trata de solucionar las angustias de un ser humano enfermo.

\section{Conflicto de intereses}

Los autores declaramos que no existen conflictos de intereses que puedan afectar los conceptos emitidos en este manuscrito.

\section{Financiación}

Para la elaboración de este ensayo, Fabián Jaimes dispuso del tiempo de la jornada laboral como profesor de la Universidad de Antioquia. El estudiante de medicina Daniel Henao no recibió financiación de ningún tipo.

\section{Referencias}

1. Evidence-Based Medicine Working Group. Evidencebased medicine. A new approach to teaching the practice of medicine. JAMA. 1992;268:2420-5.

2. Sackett DL, Rosenberg WM, Gray JA, Haynes RB, Richardson WS. Evidence based medicine: what it is and what it isn't. BMJ. 1996;312:71-2.

3. Marshall T. Evidence-based medicine. Lancet. 1995; 346:1171-2.
4. Rosenberg W, Donald A. Evidence based medicine: an approach to clinical problem-solving. BMJ. 1995;310: 1122-6.

5. Sackett D. Evidence-based medicine. Lancet. 1995;346:1171.

6. Feinstein AR, Horwitz RI. Problems in the "evidence" of "evidence-based medicine". Am J Med. 1997;103:52935.

7. Evidence-based medicine, in its place. Lancet. 1995; 346:785.

8. Grahame-Smith D. Evidence based medicine: Socratic dissent. BMJ. 1995;310:1126-7.

9. Sleigh JW. Evidence-based medicine and Kurt Godel. Lancet. 1995;346:1172.

10. Aveyard P. Evidence-based medicine. Lancet. 1995; 346: 840.

11. Bradley F, Field J. Evidence-based medicine. Lancet. 1995;346:838-9.

12. Chagla LS, McCulloch PG. Evidence-based medicine. Lancet. 1995;346:839-40.

13. Fowler PB. Evidence-based medicine. Lancet. 1995; 346:838.

14. Haynes B. Evidence-based medicine. Lancet. 1995;346: 1171.

15. Iggo N. Evidence-based medicine. Lancet. 1995;346:83940.

16. Morgan WK. Evidence-based medicine. Lancet. 1995; 346:1172.

17. Shahar E. Evidence-based medicine. Lancet.1995;346: 1172.

18. White KL. Evidence-based medicine. Lancet. 1995;346: 837-8.

19. Ellis J, Mulligan I, Rowe J, Sackett DL. Inpatient general medicine is evidence based. A-Team, Nuffield Department of Clinical Medicine. Lancet. 1995;346:40710.

20. Gill P, Dowell AC, Neal RD, Smith N, Heywood P, Wilson AE. Evidence based general practice: a retrospective study of interventions in one training practice. BMJ. 1996;312:819-21.

21. Hughes J. Evidence-based medicine. Lancet. 1995;346:839-40.

22. Norman G. Evidence-based medicine. Lancet. 1995;346:1300.

23. Canguilhem G. Augusto Comte y el "principio de Broussais". En: Lo normal y lo patológico. Primera edición. Buenos Aires: Siglo Veintiuno; 1971. p. 25-41.

24. Burgos L. Introducción a la nueva biología. Primera edición. Medellín: Biogénesis; 2003. 
25. Hubert R. Brevarios del pensamiento filosófico "Comte". Primera edición. Buenos Aires: Editorial Sudamericana; 1943.

26. Chobanian AV, Bakris GL, Black HR, Cushman WC, Green LA, Izzo JL, et al. The seventh report of the Joint National Committee on Prevention, Detection, Evaluation, and Treatment of High Blood Pressure: the JNC 7 report. JAMA. 2003;289:2560-72.
27. Canguilhem G. Lo normal y lo patológico. Primera edición. Buenos Aires: Siglo Veintiuno; 1971. p. 83-113.

28. Canguilhem G. Claude Bernard y la patología experimental. En: Lo normaly lo patológico. Primera edición. Buenos Aires: Siglo Veintiuno; 1971. p. 41-62.

29. Canguilhem G. Las concepciones de R. Leriche. En: Lo normal y lo patológico. Primera edición. Buenos Aires: Siglo Veintiuno; 1971. p. 63-71. 